\title{
OCORRÊNCIAS IATROGÊNICAS NA UTI E O ENFOQUE DE QUALIDADE
}

Katia Grillo Padilha ${ }^{1}$

Padilha KG. Ocorrências iatrogênicas na UTI e o enfoque de qualidade. Rev Latino-am Enfermagem 2001 setembro-outubro; 9(5):91-6.

Considerando as ocorrências iatrogênicas na UTI como eventos que interferem na qualidade da assistência ao doente crítico, a autora contextualiza tais ocorrências, tendo como fundamento teórico os princípios da Gestão de Qualidade Total.

PALAVRAS CHAVE: iatrogenia, gestão de qualidade, enfermagem

\section{IATROGENIC OCCURRENCES AND THE QUALITY FOCUS}

Regarding iatrogenic occurrences in ICUs as events that interfere with the quality of the care given to critical patients, the author contextualizes such occurrences by taking into account the principles of Total Quality Management as a theoretical framework.

KEY WORDS: adverse occurrence, total quality, nursing

\section{OCURRENCIAS IATROGÉNICAS Y EL ENFOQUE DE CALIDAD}

Considerando las ocurrencias adversas en la Unidad de Cuidado Intensivo (UCl) como eventos que interfieren en la calidad de la atención al paciente crítico, la autora contextualiza esas ocurrencias, utilizando como fundamentación los principios de la Gestión de Calidad Total.

PALABRAS CLAVES: ocurrencias adversas, calidad total, enfermería

\footnotetext{
${ }^{1}$ Enfermeira, Professor Associado, Departamento de Enfermagem Médico Cirúrgica da Escola de Enfermagem da Universidade de São Paulo. Endereço: Rua: Jericó, 59 - Ap. 132 - Vila Madalena - CEP: 05435-040 - São Paulo - SP - Brasil
} 
INTRODUÇÃO

A atual gerência de qualidade total nas instituições empresariais de um modo geral e nas de saúde, em particular, tem como um dos princípios básicos, 0 atendimento à clientela com 0 fornecimento de bens e de serviços que promovam a sua máxima satisfação e o mínimo, senão a ausência total de riscos e falhas que possam comprometer a qualidade e segurança pretendidas ${ }^{(1)}$. Dentro dessa perspectiva, pode-se dizer que a ocorrência de eventos iatrogênicos no decorrer da assistência ao paciente, transgride importante princípio, podendo, inclusive, colocar em risco a vida do cliente/paciente.

Mais crítica é a transposição dessas ocorrências para a Unidade de Terapia Intensiva (UTI), unidade voltada ao atendimento de pacientes graves e de alto risco, cujas condições clínicas oscilam entre limites estreitos de normalidade/anormalidade e onde pequenas mudanças orgânicas podem levar à deterioração grave na função corporal $^{(2)}$. Nela, qualquer ocorrência iatrogênica passa a ser não só indesejável, como extremamente prejudicial, fazendo emergir a questão da qualidade da assistência e o contexto na qual acontece, o que remete, inevitavelmente, para a avaliação dos serviços de saúde.

O tema qualidade nos serviços de saúde começou a ser tratado no inicio do século, nos Estados Unidos, pelo Colégio Americano de Cirurgiões, que através de padrões mínimos avaliava regularmente a qualidade do cuidado prestado aos pacientes hospitalizados. Mais tarde, a Comissão Conjunta de Acreditação de Hospitais, criada em 1951 com o objetivo de estimular o desenvolvimento técnico-administrativo dos hospitais, assumiu tais responsabilidades e passou a desenvolver novos padrões, análogos aos existentes. 0 processo de avaliação que no início era feito de maneira informal, subjetiva e com base nos conhecimentos e experiência dos avaliadores, passa por importante transformação com o desenvolvimento de métodos de avaliação estruturados e objetivos, que culminaram na auditoria médica no início dos anos $70^{(3)}$.

A partir da década de 80 , movidos por razões diversas, tais como: o elevado custo da assistência à saúde e conseqüente necessidade de redução dos gastos; 0 aumento dos processos por erro médico; a maior exigência de qualidade de cuidados por parte dos usuários; a necessidade de melhor organização dos serviços para otimizar a relação custo/benefício, a qualidade passou a ser assunto prioritário em matéria de saúde $\mathrm{d}^{(4-8)}$.

Frente a essas demandas, nos EEUU, a Comissão de Acreditação de Hospitais, em meados dos anos 80 desenvolveu extenso projeto voltado à monitorização de resultados através de indicadores, com a finalidade de ajudar organizações a melhorarem a qualidade dos cuidados oferecidos à clientela. Hoje, a existência de programas de melhoria contínua de qualidade nos hospitais americanos é condição para 0 atendimento dos pacientes inseridos nos programas sociais, sendo que cerca de $85 \%$ dos hospitais estão acreditados pela Comissão que estabelece reavaliação periódica a cada 3 anos ${ }^{(8-11)}$

Além dos Estados Unidos, verifica-se que a busca por qualidade na assistência à saúde tem sido objetivo comum em vários países do mundo, tendo como desafio oferecer a melhor assistência a um maior contingente de pessoas, a um menor custo ${ }^{(9,12-16)}$

Nesse sentido, as perspectivas de Gestão de Qualidade Total e de Melhoria Contínua de Qualidade entraram para a administração de bens com o surgimento da indústria japonesa no pós guerra, sendo que a implementação e consolidação dos conceitos modernos de qualidade são atribuídos aos especialistas americanos Deming e Juran ${ }^{(1,6,8)}$.

O conceito de Gestão de Qualidade Total envolve uma filosofia que implica no compromisso de toda a organização para a melhoria contínua de qualidade. Nesse contexto, a melhor definição de Qualidade Total é "atingir e exceder as expectativas do cliente/ consumidor, fazendo a coisa certa, de maneira certa, desde a primeira vez e melhor na seguinte"(6).

As idéias básicas a respeito do enfoque de Qualidade Total e Melhoria Contínua e que o diferencia do pensamento tradicional englobam basicamente os seguintes aspectos ${ }^{(1)}$ :

- a qualidade do produto ou serviço pode e deve ser caracterizada, contrariamente ao conceito de qualidade intangível do pensamento tradicional o que significa que, em qualquer circunstância, deve haver um conjunto de dados e afirmativas que caracterizem perfeitamente a qualidade daquele produto ou serviço;

- na organização do trabalho prevalece a orientação para o cliente/ paciente consumidor e não a ênfase nos departamentos;

- em virtude do enfoque mais global, cria-se uma parceria com o fornecedor que deverá trabalhar o seu produto também dentro de um enfoque de melhoria contínua para acompanhar os níveis de exigência crescente da empresa;

- ênfase voltada não mais à administração por objetivos, mas para a análise do processo, que permite buscar as debilidades latentes que podem vir a se transformar em perdas de qualidade;

- no pensamento de qualidade busca-se assumir atitudes pro-ativas, ou seja, valoriza-se a busca ininterrupta de falhas, de maneira preventiva, contrariamente à atitude meramente corretiva no pensamento tradicional, ou seja, a falha detectada no produto final; - busca da melhoria contínua, sem limites, inclusive com a perspectiva 
do defeito zero ou das atitudes livres de erro, mesmo que nunca atingiveis;

- ao contrário de se buscar identificar o empregado menos eficiente e eliminá-lo, busca-se conferir poder ao servidor para que ele possa tomar as decisões adequadas no momento oportuno;

- no pensamento de qualidade total as ações envolvem a instituição como um todo e não grupos de pessoas e

- ao invés de admitir como perspectiva aceitável a manutenção do status quo, investe na melhoria contínua de qualidade.

Diante deste quadro conceitual, abordar as ocorrências iatrogênicas na UTI reveste-se de particular interesse, principalmente quando considera-se que a detecção dos desvios de qualidade no decorrer da análise do processo e não do produto final implica em atitudes preventivas. Esse tipo de enfoque torna-se particularmente oportuno em "situações onde as falhas podem ser raras, mas extraordinariamente indesejáveis, como ocorre em tantas circunstâncias na produção de serviços de saúde"(1).

Um dos quadros conceituais acatados no delineamento da avaliação dos serviços de saúde propõe-se três áreas, cujo desempenho se busca avaliar: a de estrutura, processo e resultado. A primeira, corresponde aos recursos utilizados, nele incluídos os de planta física, recursos humanos, materiais, equipamentos, instrumental normativo e administrativo e as fontes de financiamento. A segunda, ou seja, a de processo, envolve as atividades relativas à utilização de recursos, nos seus aspectos quantitativos e qualitativos e a de resultado que corresponde às conseqüências da atividade do estabelecimento de saúde ou do profissional para a saúde dos indivíduos ou da população $0^{(4)}$.

Definir, compreender e quantificar indicadores apropriados na enfermagem é fundamental para avaliar a qualidade dos serviços, não só pelas exigências da Comissão de Acreditação, como também em função das exigências do público frente aos serviços de saúde que the são oferecidos ${ }^{(11)}$. Para a autora ${ }^{(11)}$, indicadores clínicos são definidos como medidas quantitativas que podem ser usadas como guias para monitorar e avaliar a qualidade de importantes cuidados ao paciente. Afirma que indicadores não devem ser confundidos como medidas diretas da qualidade, mas como marcadores que podem identificar áreas que necessitam de avaliação mais acurada. Considerando as áreas de avaliação propostas por Donabedian, a autora enfatiza que indicadores de resultados são aqueles que acessam exatamente o resultado final do cuidado ao paciente, seja ele desejável ou indesejável.

Tendo por base a teoria da melhoria continua de qualidade e a avaliação das áreas de estrutura, processo e resultado constatase que na vigência de problemas relacionados à qualidade do cuidado, falhas subjacentes ao sistema, tais como falta de liderança, não definição de objetivos e mesmo estratégias deficientes de trabalho devem ser procuradas. Assim, a monitorização das atividades, seu seguimento, avaliação e intervenções devem ser realizadas num ciclo incessante e contínuo de investigação e mudança. Porém, para saber o que está errado, o primeiro passo é identificar os eventos adversos ou potencialmente adversos numa tentativa de se elucidar as causas subjacentes e os fatores contribuintes ${ }^{(17)}$.

\section{OCORRÊNCIAS IATROGÊNICAS NA UTI}

Buscando investigar os incidentes adversos ou ocorrências iatrogênicas no decorrer da assistência em uma UTI geral, sob o enfoque da melhoria contínua de qualidade, um grupo de pesquisadores investigou os incidentes críticos relatados no período de um ano por médicos e enfermeiros intensivistas. Obtiveram relatos de 390 incidentes, dos quais resultaram uma morte, 86 complicações severas e 88 complicações de menor gravidade. Os incidentes encontrados, em ordem decrescente, foram associados a equipamentos, medicamentos, procedimentos e outros da esfera administrativa $^{(18)}$.

Autores diversos, de diferentes países, têm investido na busca de maior conhecimento sobre esses eventos, tendo por finalidade a sua monitorização e intervenção.

Dentro de um programa de monitorização de incidentes críticos, estudo de abrangência nacional, realizado em 7 UTIs australianas, identificou 610 incidentes, com maior freqüência daqueles relacionados à drogas ( $28 \%$ ), seguido dos procedimentos $(23 \%)$, ambiente do paciente $(21 \%)$, vias aéreas $(20 \%)$ e problemas administrativos (9\%). Os autores relatam que nenhum efeito ou apenas os de menor severidade acometeram os pacientes nos incidentes estudados ${ }^{(5)}$.

Também nesta linha de investigação e integrado ao Estudo da Monitorização de Incidentes na Austrália e Nova Zelândia, desenvolvido pela Fundação para a Segurança dos Pacientes, foram estudados 2000 relatos de incidentes críticos apresentados por anestesistas de 90 hospitais ${ }^{(19)}$. Nesta investigação os pesquisadores apontam as vantagens da utilização de um sistema estruturado de registro de situações, de caráter anônimo, uma vez que permite a identificação dos fatores contribuintes para a ocorrência do incidente, bem como 0 acesso às medidas de correção sugeridas. Como resultados, verificaram que do total de relatos, a maior freqüência dos eventos ocorreu durante a manutenção da anestesia (48\%). A categoria mais comum de incidentes envolveu a desconexão do 
circuito respiratório e no que se refere às conseqüências dos incidentes, observaram que não houve nenhum resultado negativo em $44 \%$ dos casos.

Também na China, estudo sobre incidentes críticos relatados em uma UTI de adultos como parte do programa de Melhoria Contínua de Qualidade, no período de 3 anos, analisou 281 relatos que apontaram como incidentes mais comuns aqueles relacionados ao manuseio das vias aéreas e dos drenos, tubos, cateteres. Como conseqüência desses incidentes, nenhuma mudança fisiológica foi observada em $54 \%$ dos relatos ${ }^{(20)}$.

No Brasil, estudo prospectivo com 517 pacientes internados na UTI de um hospital universitário, durante 18 meses, constatou que 95 deles sofreram algum tipo de iatrogenia, o que correspondeu a 18,4 \% das internações na Unidade. Os 95 pacientes sofreram 120 complicações, correspondendo, portanto, a mais do que uma por paciente. Os resultados indicaram que os métodos invasivos antes da internação na UTI foram as causas mais freqüentes das ocorrências adversas enquanto que, durante a internação, drogas e métodos invasivos foram equivalentes ${ }^{(21)}$.

A incidência de eventos iatrogênicos na UTI, embora investigada por alguns pesquisadores, tem sido variável e passível de controvérsias , em função do uso de diferentes metodologias que dificultam análises comparativas.

Apesar disso, extensa investigação realizada no Estado de Nova York em 1984, em resposta ao aumento dos processos de malpractice contra os profissionais de saúde, possibilitou a análise de aproximadamente 30 mil prontuários selecionados de 51 hospitais. Demonstrou-se com esse estudo que eventos adversos ocorreram em $3,7 \%$ das admissões, dos quais $25 \%$ foram atribuídos à negligência. Concluíram os autores que existe uma substancial quantidade de injúrias acometendo o paciente no hospital e que apreciável quantidade delas é resultante de um cuidado abaixo dos padrões profissionais estabelecidos para a prática ${ }^{(22-23)}$.

Replicando tal estudo, foi realizada a revisão de 14 mil prontuários de pacientes admitidos em 28 hospitais da Austrália ${ }^{(24)}$. Observou-se que $16,6 \%$ das admissões foram associadas a um evento adverso, que resultou em incapacidade ou aumento da permanência do doente no hospital. Diante de um achado expressivamente maior que 0 americano, os pesquisadores discutem os dados encontrados e levantam questionamentos sobre as causas que estariam levando a índices tão diferentes entre os dois países.

Também estudo feito na década de 80 sobre as iatrogenias ocorridas durante um período de 5 meses, em um hospital universitário ${ }^{(25)}$ apontou incidência ainda maior, ou seja, $36 \%$ dos 815 pacientes internados consecutivamente sofreram algum tipo de doença iatrogênica. Em 9\% do total de admitidos tais ocorrências foram consideradas graves e em $2 \%$ acredita-se tenham contribuído para a morte dos pacientes
Analisando-se os dados apresentados na literatura, verificase que o fator humano é apontado como o principal agente causador das ocorrências iatrogênicas ou incidentes críticos ${ }^{(20,26-29)}$. No entanto, embora grande porcentagem dos problemas tenha o componente humano envolvido, sua contribuição global para muitos problemas deve ser analisada.

Dentro do princípio da melhoria contínua de qualidade, a análise dos mecanismos pelos quais produtos "pobres" são produzidos levou à conclusão que $85 \%$ dos problemas são relacionados ao sistema e que pequena porcentagem é resultante da deficiente performance ou erro do ser humano, exclusivamente. Embora um ato individual possa ser o precursor de um acidente, muitas vezes é apenas o agente final de um processo mais abrangente ${ }^{(14)}$

$\mathrm{Na}$ realidade brasileira, a questão da estrutura, diferentemente do que acontece nos Estados Unidos e na Austrália, onde apresentam elevados padrões e encontram-se sob avaliação periódica ${ }^{(4,17,30)}$ apresenta problemas consideráveis que precisam ser levados em conta quando se investigam as ocorrências iatrogênicas.

A falta de recursos para o atendimento à saúde, a ineficiência dos serviços, as condições precárias de planta física, recursos humanos escassos e equipamentos obsoletos, são aspectos muito discutidos nos dias de hoje, sobretudo pelos órgãos fiscalizadores profissionais ${ }^{(31-36)}$

Assim, embora os estudos anteriormente referidos apontem para a questão do fator humano nas ocorrências, condições específicas do atendimento à saúde no contexto nacional, levam a investir também na investigação dos aspectos estruturais existentes. O conhecimento das condições de trabalho dos profissionais, bem como as atividades desenvolvidas em duplas jornadas e as condições de saúde, merecem igual atenção, pois podem precipitar o desencadeamento de ocorrências iatrogênicas, interferindo na qualidade da assistência ${ }^{(37)}$.

Sendo a UTI uma unidade complexa, de intenso dinamismo, que congrega diferentes profissionais e onde as tomadas de decisão devem ser prontas e precisas, espera-se estrutura adequada como suporte para o desenvolvimento de uma assistência segura ao doente crítico.

Nesse contexto, em que pesem as implicações ético-legais envolvidas no problema em questão, acredita-se que a análise das ocorrências iatrogênicas na UTI face à abrangência do enfoque de Qualidade Total e de Melhoria Contínua de Qualidade abre importantes perspectivas para a prevenção dessas ocorrências. Mais do que achar culpados para punir, apenas o olhar crítico e investigativo sobre cada falha existente no sistema pode apontar as lacunas que precisam ser sanadas visando ao benefício do próprio doente e de todos os envolvidos no processo de assistência à saúde. 


\section{REFERÊNCIAS BIBLIOGRÁFICAS}

1. Azevedo AC. O futuro dos hospitais e a gestão da qualidade. Rev Paul Hosp 1992 maio/dezembro; 40(5/12):53-9.

2. Hoytt JW, Harvey MA, Axon DC. The critical care unit: design recommendations and standards. In: Shoemaker WC. Textbook of critical care. Philadelphia: W.B. Saunders; 1995. p.7-14.

3. Basset SS. Quality assurance-quality improvement. In: Koch MW, Fairly TM. Integrated quality management. St. Louis (MO): Mosby Year; 1993. p. 56-72.

4. Azevedo AC. Avaliação de desempenho de serviços de saúde. Rev Saúde Pública 1991 janeiro; 25:64-71.

5. Beckmann U, Balwin I, Hart GK, Runciman WB. The australian incident monitoring study in intensive care: AIMS-ICU an analysis of the first year of reporting. Anaesth Intens Care 1996 June; 24(3):3209.

6. Masters F, Schmele JA. Total quality managememt: an idea whose time has come. J Nurs Qual Assur 1991 July; 5(4):7-16.

7. Mezomo JC. O desafio e o sucesso da qualidade. Hosp Adm Saúde 1991 junho; 15(6):241-3.

8. Mezomo JC. Em busca da qualidade nos serviços. Hosp Adm Saúde 1992 fevereiro; 16(2): 55-8.

9. Wooton P. Assistência médica e os cortes nos custos da saúde nos Estados Unidos. Jornal CFM 1998 janeiro; 12(89): 15-6.

10. Human D. Empresas multinacionais de saúde e o trabalho médico no mundo globalizado. Jornal CFM 1998 janeiro;12(89):17-8.

11. Williams AD. Development and application of clinical indicators for nursing. J Nurs Care Qual 1991 October; 6(1):1-5.

12. Berwick DM. Continuous improvement as an ideal in health care. N Engl J Med 1989 January; 320(1):53-6.

13. Direito à saúde: responsabilidade política e social do estado/ Entrevista/Jornal CFM 1997 outubro; 10(86):22-3.

14. Runciman WB, Selle A, Webb RK, Willianson JA, Currie M, Morgan $C$ et al. Errors, incidents and accidents in anaesthetic practice. Anaesth Intens Care 1993 October; 21(5):506-19.

15. Saúde engessada. /Editorial/ Jornal CFM 1992 setembro; 7(27):2. 16. Saúde nos Estados./Entrevista/ Jornal CFM 1998 fevereiro; 12(90): 22-3.

17. Beckmann U, West LF, Groombridge GJ, Balwin I, Hart GK, Hart GK et al. The australian incident monitoring study in intensive care: AIMS-ICU. The Development and evaluation of an incident reporting system in intensive care. Anaesth Intens Care 1996 June; 24(3):3149 .

18. Hart GK, Baldwin I, Gutteridge G, Ford J. Adverse incident reporting in Intensive care. Anaesth Intens Care 1994 October; 22(5):556-61.
19. Webb RK, Currie M,Morgan CA, Williamson JA, Mackay P, Russel WJ et al. The australian incident monitoring study: an analysis of 2000 incident reports. Anaesth Intens Care 1993 October; 21(5):5208.

20. Buckley TA, Short TG, Rowbottom YM, Oh TE. Critical incident reporting in the intensive care unit. Anaesthesia 1997 January; 52(1):403-9.

21. David CM, Vargas SSM, Hoirisch S. Doenças iatrogênicas em terapia intensiva. Folha Med 1984 agosto; 89(2):107-12.

22. Brennan TA, Leape LL, Laird NM, Hebert L, Localio AR, Lawthers $A G$ et al. Incidence of adverse events and negligence in hospitalized patients: results of the Harvard medical practice study I. N Engl J Med 1991 February; 324(2):370-6.

23. Leape LL, Brennan TA, Laird N, Lawthers AG, Localio AR, Barnes BA et al. The nature of adverse events in hospitalized patients: results of the Harvard medical practice study II. N Engl J Med 1991 February; 324(6):377-84.

24. Wilson RM, Runciman WB,Gibberd RW, Harrison BT, Newby L, Hamilton JD. The quality in australian health care study. Med J Aust 1995 November; 163(6):458-71.

25. Steel K, Gertman PM, Crescenzic, Anderson J. latrogenic illness on a general medical service at a university hospital. N Engl J Med 1981 March; 304(12):638-42.

26. Cooper JB, Newbower RS, Kitz RJ. An analysis of major errors and equipment failures in anesthesia management: considerations for prevention and detection. Anesthesiology 1984 January; 60(1):3442.

27. Donchin Y, Gopher D, Olin M, Badihi Y, Biesky M, Sprung CL et al. A look into the nature and causes of human errors in the intensive care unit. Crit Care Med 1995 February; 23(2): 294-300.

28. Pierin AMG, Ide CAC, Padilha KG, Marcowiski W. latrogenia em enfermagem. Rev Esc Enfermagem USP 1983 agosto; 17(2):11925.

29. Wright D, Mackenzie SJ, Buchan I, Cairns CS, Price LE. Critical incidents in the intensive therapy unit. Lancet 1991 September; 38(14):676-8.

30. Rutstein DD, Berenberg, W, Chalmers, TC, Child CG, Fishman AP, Perrin EB. Measuring the quality of medical care. N Engl J Med 1976 March; 294(11):582-8.

31. Atendimento médico: perspectivas para a virada do milênio. Ser médico 1998 janeiro/março; 1(2):23-8.

32. Entidades concluem radiografia do país. Jornal CFM 1990 maio; 5(19):6-7.

33. A escada orçamentária/Entrevista/. Médicos 1998 julho/agosto; 1(3):6-11. 
34. Ferraz MB. Sistema de saúde brasileiro: suas limitações e seus desafios. Ser médico 1997 janeiro/março;1(1):10-3.

35. Fiscalização dos estabelecimentos de saúde e equipe multiprofissional. Jornal Cremesp 1992 março; 12(70):7.
36. Hospitais universitários pedem socorro. Jornal CFM 1997 novembro; 10(87):21.

37. Padilha KG. Des-cuidar: As representações sociais dos enfermeiros de UTI sobre as ocorrências iatrogênicas de enfermagem. [dissertação]. São Paulo (SP): Escola de Enfermagem/USP; 1994. 